\title{
Choosing the Adaptive Cardiac Phase for Assessing Cardiac Dimensions Using Cardiac Computed Tomography for Heart Disease
}

\author{
Li Wang \\ The Affiliated Hospital of Southwest Medical University \\ Jin-Rong Zhou \\ The Affiliated Hospital of Southwest Medical University \\ Dong Chen \\ The Affiliated Hospital of Southwest Medical University \\ Yu-Jiao Deng \\ The Affiliated Hospital of Southwest Medical University \\ Jing Chen ( $\nabla$ yzqdcj@126.com ) \\ Deparment of Radiology, the Affiliated Hospital of Southwest Medical University
}

\section{Research Article}

Keywords: chamber dimension, wall thickness, cardiac phase, cardiac computed tomography

Posted Date: August 19th, 2021

DOI: https://doi.org/10.21203/rs.3.rs-814699/v1

License: (a) This work is licensed under a Creative Commons Attribution 4.0 International License. Read Full License 


\section{Abstract}

Background Choosing a suitable cardiac cycle to measure cardiac chamber dimensions and wall thickness can be a more accurate assessment of cardiovascular disease.

Methods Cardiac CT was performed on 137 patients for suspected coronary disease. The parameters of left atrium (LA), left ventricle (LV), right atrium (RA), and right ventricle (RV), as well as the wall thickness of LV were measured in different cardiac phases. The general linear mixed model was used to analyze differences in different phases and the correlation between these parameters and traditional risk factors. ROC analysis was performed to estimate LA enlargement.

Results The dimensions of LA, RA, and LV wall thickness achieved the maximum at the phase of $35-45 \%$, and those of LV and RV, at $95-5 \%$. Whereas, the changes of LA-B (antero-posterior diameter), LV-D1 (basal dimension), RA-B (minor dimension) and RV-D2 (mid cavity dimension) were relatively more stable during the cardiac cycle. The maximum LA-B diameter(95\%Cl 36.92,38.48mm), LV-D1 diameter(95\%Cl 44.36,45.83mm), RA-B diameter $(95 \% \mathrm{Cl} 48.75,50.61 \mathrm{~mm})$, and RV-D2 diameter(95\% Cl 30.83,32.84mm) and the maximum interventricular septum thickness( $95 \% \mathrm{Cl}$ $10.79,11.51 \mathrm{~mm}$ ) was acquired. Heart rate (HR) and smoking were potential indicators of LVD2 (mid cavity dimension), while HR and LV myocardial mass were potential indicators of LVD3 (apical-basal dimension). In phase $45 \%$, the cut-off value of LA-B was $37.12 \mathrm{~mm}$ has high sensitivity of $90.9 \%$ for predicting LA enlargement.

Conclusion Cardiac chamber dimensions and wall thickness vary with the cardiac phase. Choosing the adaptive cardiac phase for evaluating these parameters obtained by cardiac CT could provide a more accurate clinical measurement.

Trial registration retrospectively registered.

\section{Introduction}

The heart is a hollow organ at the center of the circulatory system. Rhythmic contractions and dilations by the contraction of myocardial cells pump the blood. Cardiac remodeling is an important pathological process in the occurrence and development of various heart diseases, not only changes in the diameter of the cardiac chambers but also changes in the thickness of the ventricular wall. These conditions can remain clinically silent until late in their progression. Therefore, it is important to recognize changes in cardiac chamber dimensions and wall thickness early in the course of these diseases. In addition, assessing the dimensions of the cardiac chambers is useful for diagnosis of cardiac disease, risk stratification, and therapeutic decision making. For example, left atrial (LA) enlargement is associated with an increased risk of adverse cardiovascular events, such as atrial fibrillation (AF), myocardial infarction, congestive heart failure, and stroke[1, 2]. Left ventricular (LV) enlargement is associated with an array of cardiac pathologies, including cardiomyopathy, ischemia, and valvular heart disease. Moreover, right heart function and diameter are independent determinants of clinical status and prognosis in many congenital and acquired disease states. Therefore, obtaining accurate diameters of cardiac chambers and wall thickness are crucial to clinical decision-making and follow-up for cardiac diseases.

It is well-known that cardiac chamber dimensions and LV wall thickness change with the cardiac cycle, and many studies have confirmed that cardiac diameters differ in systole and diastole[3,4]. However, the cardiac diameters are usually measured during a random phase of the cardiac cycle, and the result might be the largest or the smallest or in the between. This can especially affect the choice of ablation method for patients with AF and the prognosis after ablation. The dimension of the LA, especially the maximum diameter of the antero-posterior diameter (LA-B) is used as a parameter of ablation method and the prognosis after ablation. Some research has shown that cryoballoon (CB) ablation may be recommended for patients with LA-B $\otimes 4 \mathrm{~cm}$, while radiofrequency (RF) would be more appropriate for patients with LA-B $>4 \mathrm{~cm}[5]$. Therefore, it is also crucial to choose an accurate cardiac phase to measure the dimensions of the cardiac chamber. Echocardiography is the most commonly used noninvasive modality for evaluating the size of the cardiac chambers, but it is heavily dependent on the sonographer's skills and has poor repeatability. Cardiac MRI is considered the reference standard for evaluating cardiac size and function, but it is costly and time-consuming[6]. Moreover, cardiac MRI is rarely performed as an initial investigation for evaluating cardiac size. Multi-slice spiral computed tomography (CT) combined with retrospective electrocardiography (ECG) gating could provide heart images with high time resolution and reconstruct multi-phase images of the whole cardiac cycle[7, 8]. It can comprehensively evaluate lesions that cause the heart to enlarge for many reasons, and is emerging as a promising tool with respect to quantifying chamber volumes and cardiac structure $[9,10]$. Therefore, it is recommended to use the technology of ECG gated CT acquisition for evaluating the diameter of cardiac chamber(especially LA-B) accurately instead of motion artifacts.

In recent years, many scholars have focused cardiac chamber dimensions changed just on end-systole and end-diastole phase[3, 4]. Moreover, different researchers have set different time points for systole and diastole in the cardiac cycle. This makes the assessment of the cardiac chamber dimensions inconsistent in the cardiac phase and results in different dimensions for the same structure,especially different heart rate. Therefore, the aim of our study was to assess the dynamic changes of the normal cardiac chamber dimensions and wall thickness in ten cardiac phases,which is used to compare abnormal cardiac disease. While we obtained a threshold for judging left atrium enlargement in CT image.

\section{Methods}


This study was conducted in accordance with the the Declaration of Helsinki (as was revised in 2013), and an application for the exemption of patients' informed consent was approved by the Institutional Review Board of our hospital, due to the retrospective nature of the study. So all written informed consent forms are waived.

\subsection{Patient selection}

Two hundred consecutive patients underwent cardiac CT scans for suspected coronary disease between August and December 2019. Patients with normal cardiac chamber dimensions or LA enlargement on echocardiography (within three months of the CT study) and who were at least 18 years of age were enrolled. Exclusion criteria included patients with severe arrhythmia, pacemakers, cardiovascular surgical procedures, known heart disease and motion artifact. Patients with structural or functional abnormalities of the heart detected by echocardiography were also excluded.

\subsection{CT scanning protocol}

All CT angiography (CTA) scans were acquired using a 256-slice scanner (Philips Brilliance iCT; Philips Medical Systems, Cleveland, OH, USA) with retrospective ECG-gating. The scanning range is from the bifurcation of the pulmonary artery to the level of the diaphragm with a thickness of $0.5 \mathrm{~cm}$. The scan is automatically triggered by setting the region of interest in the descending aorta (the threshold value was set as $250 \mathrm{Hounsfield} \mathrm{units} \mathrm{[Hu]).}$ A high-pressure syringe was used to inject $75-85 \mathrm{~mL}$ of contrast agent (lopamidol $370 \mathrm{mg} / \mathrm{mL}$ ) through the elbow vein. Subsequently, $40 \mathrm{~mL}$ of physiological saline was injected at the same rate with a flow rate of $5.0-5.5 \mathrm{~mL} / \mathrm{s}$. We used $120 \mathrm{kv}$ for patients with body weight $\geq 75 \mathrm{~kg}, 100 \mathrm{kV}$ for patients with body weight $<75 \mathrm{~kg}, 1200 \mathrm{mAs}$ /slice for patients with body mass index (BMI) $\geq 24$, and $900 \mathrm{mAs} /$ slice for patients with $\mathrm{BMI}<24$.

\subsection{Image analysis}

Multi-phase reconstructions were done from 5-95\% in increments of $10 \%$ on a 256 -slice spiral CT scanning workstation. The reconstruction vision of 10 cardiac cycles was extended to the entire thorax. All images were transferred to a post-processing workstation (Philips Intelli Space Portal system) and loaded into the cardiac viewer application. Two experienced observers (both with three years of experience in the interpretation of cardiac CT images) who were blinded to the echocardiographic data independently reviewed the reconstructed CT images on a dedicated post-processing workstation.

The left atrial maximum transverse diameter (LA-A) and antero-posterior diameter (LA-B) were to be traced manually on an image showing the right inferior pulmonary vein insertion[11]. The thoracic vertebral diameter was measured at the same level[12]. The left atrial-vertebral ratio (LAssVR) was then obtained by dividing the LA diameter by the vertebral diameter (Fig. 1A). The left ventricular basal dimension (LVD1), mid cavity dimension (LVD2) and apical-basal dimension (LVD3) in different cardiac phases were measured in the long-axis view of LV[13] (Fig. 1D). LV wall

thickness(including 16 segmentations) at the basal, mid, and apical segments in different cardiac phases were measured in the short-axis view of the LV (Fig. 1C). The right atrial major dimension (RA-A), minor dimension (RA-B), right ventricular basal dimension (RVD1), mid cavity dimension (RVD2), and apical-basal dimension (RVD3) in different cardiac phases were measured in the apical four-chamber multiplanar reconstruction view[14, 15] (Fig. 1B).

\subsection{Statistical analysis}

The statistical software package R (Version 3.6.1; R Core Team, 2019) was used to perform analyses. Qualitative data were expressed by frequency and percentage, while quantitative data were expressed by mean \pm standard deviation. Differences in wall thickness and cardiac chamber dimensions between cardiac cycles were detected using linear mixed model. The relationship between LV-D2/LV-D3 and clinical characteristics were also estimated by linear mixed model. ROC analyze together with area under curve, sensitivity and specificity were used to estimate the diagnosis value of single and combined parameters for left atrial enlargement. Intra- and inter-observer variability for reproducibility were assessed using intraclass correlation coefficient (ICCs). The significance level was set at $p<0.05$.

\section{Results}

\subsection{Baseline characteristics}

We enrolled a total of 126 subjects (65 males and 61 females, mean age 55 years, range 47-77 years) with normal chamber dimensions and 118 subjects (61 men and 57 women, mean age 54 years, range 30-77 years) with normal wall thickness, 11 patients( 6 men and 5 women, mean age 58 years, range 55-74 years) with LA enlargement. Table 1 shows the clinical characteristics of the study population. 
Table 1

General clinical characteristics

\begin{tabular}{|c|c|c|c|}
\hline Characteristics & $\begin{array}{l}\text { NCD group } \\
(N=126)\end{array}$ & $\begin{array}{l}\text { NWT group } \\
(N=118)\end{array}$ & $\begin{array}{l}\text { LAE group } \\
(\mathrm{N}=11)\end{array}$ \\
\hline Age(years) & $55.52 \pm 11.38$ & $54.89 \pm 11.18$ & $64.5 \pm 7.74$ \\
\hline Sex(male;n,\%) & $65(51.6 \%)$ & $61(51.7 \%)$ & $6(54.5 \%)$ \\
\hline $\mathrm{BMI}(\mathrm{kg} / \mathrm{m} 2)$ & $23.95 \pm 2.68$ & $24.17 \pm 2.70$ & $23.63 \pm 2.29$ \\
\hline $\mathrm{BSA}(\mathbb{\nabla})$ & $1.65 \pm 0.16$ & $1.65 \pm 0.15$ & $1.67 \pm 0.17$ \\
\hline Smoking(n,\%) & $30(23.8 \%)$ & $29(24.6 \%)$ & $2(18.2 \%)$ \\
\hline Drinking(n,\%) & $22(17.5 \%)$ & $21(17.8 \%)$ & $1(9 \%)$ \\
\hline Hypertension(n,\%) & $34(27.0 \%)$ & $30(25.4 \%)$ & $5(45.5 \%)$ \\
\hline Hyperglycemia(n,\%) & $15(11.9 \%)$ & $15(12.7 \%)$ & $1(9 \%)$ \\
\hline Hyperlipemia(n,\%) & $32(25.4 \%)$ & $31(26.3 \%)$ & $3(27.3 \%)$ \\
\hline HR(Beats/min) & $62.98 \pm 8.67$ & $62.98 \pm 8.72$ & $64.64 \pm 20.5$ \\
\hline LVMM(g) & $111.07 \pm 26.75$ & $110.39 \pm 24.09$ & $117.34 \pm 33.14$ \\
\hline
\end{tabular}

\subsection{LA parameters in different cardiac cycles}

Table 2 list the mean $\pm s d$ of LA-A, LA-B, and LAVR in different cardiac phases, Fig. 2A shows the change trends. For LA-A, LA-B, and LAVR, the maximum and minimum values were observed in the $35-45 \%$ phase and $5 \%$ phase, respectively. The maximum values of $L A-A$ and $L A-B$ were 67.03 $\pm 5.39 \mathrm{~mm}$ and $37.70 \pm 4.40 \mathrm{~mm}$ respectively. The minimum value of LA-B was $29.54 \pm 4.43 \mathrm{~mm}$. The variation of LA-B in cardiac cycles was of statistical significance, LA-A and LAVR were not. Compared with LA-A, LA-B changes more stably during the cardiac cycle. 
Table 2

Cardiac chamber dimensions in different cardiac cycles

\begin{tabular}{|c|c|c|c|c|c|c|c|c|c|c|c|}
\hline Phase & LA-A & LA-B & LAVR & LV-D1 & LV-D2 & LV-D3 & RA-A & RA-B & RV-D1 & RV-D2 & RV-D3 \\
\hline $5 \%$ & $\begin{array}{l}54.26 \pm \\
6.28\end{array}$ & $\begin{array}{l}29.54 \pm \\
4.43\end{array}$ & $\begin{array}{l}1.77 \pm \\
0.24\end{array}$ & $\begin{array}{l}45.09 \pm \\
4.16\end{array}$ & $\begin{array}{l}50.44 \pm \\
4.73\end{array}$ & $\begin{array}{l}77.36 \pm \\
8.36\end{array}$ & $\begin{array}{l}39.46 \pm \\
5.72\end{array}$ & $\begin{array}{l}36.95 \pm \\
4.84\end{array}$ & $\begin{array}{l}42.13 \pm \\
5.19\end{array}$ & $\begin{array}{l}31.28 \pm \\
5.19\end{array}$ & $\begin{array}{l}74.35 \pm \\
7.96\end{array}$ \\
\hline $15 \%$ & $\begin{array}{l}56.24 \pm \\
5.90\end{array}$ & $\begin{array}{l}30.71 \pm \\
4.69\end{array}$ & $\begin{array}{l}1.84 \pm \\
0.24\end{array}$ & $\begin{array}{l}43.80 \pm \\
4.69\end{array}$ & $\begin{array}{l}45.99 \pm \\
6.16\end{array}$ & $\begin{array}{l}74.75 \pm \\
7.04\end{array}$ & $\begin{array}{l}42.24 \pm \\
5.51\end{array}$ & $\begin{array}{l}40.55 \pm \\
5.29\end{array}$ & $\begin{array}{l}41.70 \pm \\
5.24\end{array}$ & $\begin{array}{l}29.68 \pm \\
4.95\end{array}$ & $\begin{array}{l}71.06 \pm \\
7.26\end{array}$ \\
\hline $25 \%$ & $\begin{array}{l}61.49 \pm \\
5.65\end{array}$ & $\begin{array}{l}33.71 \pm \\
4.52\end{array}$ & $\begin{array}{l}1.90 \pm \\
0.30\end{array}$ & $\begin{array}{l}41.06 \pm \\
4.67\end{array}$ & $\begin{array}{l}37.98 \pm \\
6.69\end{array}$ & $\begin{array}{l}66.57 \pm \\
7.47\end{array}$ & $\begin{array}{l}44.55 \pm \\
5.50\end{array}$ & $\begin{array}{l}45.24 \pm \\
5.45\end{array}$ & $\begin{array}{l}38.60 \pm \\
4.97\end{array}$ & $\begin{array}{l}25.90 \pm \\
4.87\end{array}$ & $\begin{array}{l}65.21 \pm \\
7.32\end{array}$ \\
\hline $35 \%$ & $\begin{array}{l}65.74 \pm \\
5.60\end{array}$ & $\begin{array}{l}36.52 \pm \\
4.44\end{array}$ & $\begin{array}{l}1.92 \pm \\
0.29\end{array}$ & $\begin{array}{l}38.64 \pm \\
4.85\end{array}$ & $\begin{array}{l}30.89 \pm \\
7.23\end{array}$ & $\begin{array}{l}58.94 \pm \\
8.38\end{array}$ & $\begin{array}{l}45.37 \pm \\
5.50\end{array}$ & $\begin{array}{l}48.62 \pm \\
5.27\end{array}$ & $\begin{array}{l}36.13 \pm \\
5.35\end{array}$ & $\begin{array}{l}23.17 \pm \\
4.99\end{array}$ & $\begin{array}{l}60.11 \pm \\
7.55\end{array}$ \\
\hline $45 \%$ & $\begin{array}{l}67.03 \pm \\
5.39\end{array}$ & $\begin{array}{l}37.70 \pm \\
4.40\end{array}$ & $\begin{array}{l}1.91 \pm \\
0.29\end{array}$ & $\begin{array}{l}37.55 \pm \\
5.43\end{array}$ & $\begin{array}{l}31.15 \pm \\
7.97\end{array}$ & $\begin{array}{l}58.77 \pm \\
8.36\end{array}$ & $\begin{array}{l}45.58 \pm \\
5.18\end{array}$ & $\begin{array}{l}49.68 \pm \\
5.26\end{array}$ & $\begin{array}{l}36.00 \pm \\
5.89\end{array}$ & $\begin{array}{l}23.44 \pm \\
5.40\end{array}$ & $\begin{array}{l}58.63 \pm \\
7.33\end{array}$ \\
\hline $55 \%$ & $\begin{array}{l}63.99 \pm \\
6.22\end{array}$ & $\begin{array}{l}35.51 \pm \\
5.07\end{array}$ & $\begin{array}{l}1.90 \pm \\
0.29\end{array}$ & $\begin{array}{l}40.16 \pm \\
5.57\end{array}$ & $\begin{array}{l}39.88 \pm \\
8.05\end{array}$ & $\begin{array}{l}66.16 \pm \\
9.53\end{array}$ & $\begin{array}{l}44.90 \pm \\
5.68\end{array}$ & $\begin{array}{l}45.27 \pm \\
5.77\end{array}$ & $\begin{array}{l}39.07 \pm \\
6.18\end{array}$ & $\begin{array}{l}27.22 \pm \\
5.85\end{array}$ & $\begin{array}{l}63.02 \pm \\
8.29\end{array}$ \\
\hline $65 \%$ & $\begin{array}{l}61.22 \pm \\
5.91\end{array}$ & $\begin{array}{l}33.91 \pm \\
4.65\end{array}$ & $\begin{array}{l}1.88 \pm \\
0.28\end{array}$ & $\begin{array}{l}41.84 \pm \\
4.47\end{array}$ & $\begin{array}{l}44.56 \pm \\
6.10\end{array}$ & $\begin{array}{l}70.55 \pm \\
7.51\end{array}$ & $\begin{array}{l}45.01 \pm \\
5.22\end{array}$ & $\begin{array}{l}42.62 \pm \\
5.25\end{array}$ & $\begin{array}{l}41.15 \pm \\
6.11\end{array}$ & $\begin{array}{l}29.36 \pm \\
6.04\end{array}$ & $\begin{array}{l}65.92 \pm \\
8.14\end{array}$ \\
\hline $75 \%$ & $\begin{array}{l}60.52 \pm \\
5.66\end{array}$ & $\begin{array}{l}33.94 \pm \\
4.33\end{array}$ & $\begin{array}{l}1.85 \pm \\
0.26\end{array}$ & $\begin{array}{l}42.62 \pm \\
4.17\end{array}$ & $\begin{array}{l}45.42 \pm \\
6.34\end{array}$ & $\begin{array}{l}71.59 \pm \\
7.39\end{array}$ & $\begin{array}{l}44.56 \pm \\
5.47\end{array}$ & $\begin{array}{l}41.96 \pm \\
4.84\end{array}$ & $\begin{array}{l}41.20 \pm \\
5.92\end{array}$ & $\begin{array}{l}29.92 \pm \\
5.96\end{array}$ & $\begin{array}{l}67.28 \pm \\
7.69\end{array}$ \\
\hline $85 \%$ & $\begin{array}{l}59.69 \pm \\
5.93\end{array}$ & $\begin{array}{l}33.97 \pm \\
4.22\end{array}$ & $\begin{array}{l}1.82 \pm \\
0.26\end{array}$ & $\begin{array}{l}43.40 \pm \\
4.19\end{array}$ & $\begin{array}{l}46.56 \pm \\
5.71\end{array}$ & $\begin{array}{l}72.86 \pm \\
7.10\end{array}$ & $\begin{array}{l}43.76 \pm \\
5.48\end{array}$ & $\begin{array}{l}41.00 \pm \\
4.83\end{array}$ & $\begin{array}{l}41.40 \pm \\
5.79\end{array}$ & $\begin{array}{l}30.22 \pm \\
5.71\end{array}$ & $\begin{array}{l}68.54 \pm \\
7.81\end{array}$ \\
\hline $95 \%$ & $\begin{array}{l}55.62 \pm \\
6.07\end{array}$ & $\begin{array}{l}31.53 \pm \\
4.75\end{array}$ & $\begin{array}{l}1.80 \pm \\
0.38\end{array}$ & $\begin{array}{l}44.91 \pm \\
4.07\end{array}$ & $\begin{array}{l}49.66 \pm \\
5.17\end{array}$ & $\begin{array}{l}76.37 \pm \\
7.25\end{array}$ & $\begin{array}{l}39.44 \pm \\
5.45\end{array}$ & $\begin{array}{l}36.77 \pm \\
5.02\end{array}$ & $\begin{array}{l}41.95 \pm \\
5.56\end{array}$ & $\begin{array}{l}31.83 \pm \\
5.69\end{array}$ & $\begin{array}{l}73.50 \pm \\
8.22\end{array}$ \\
\hline $\mathrm{t}$ & 1.858 & 6.601 & -0.317 & 2.938 & 6.699 & 3.700 & 1.624 & -4.603 & 4.885 & 7.889 & 0.669 \\
\hline $\begin{array}{l}p \text { - } \\
\text { value }\end{array}$ & 0.063 & $<0.001$ & 0.752 & 0.003 & $<0.001$ & $<0.001$ & 0.105 & $<0.001$ & $<0.001$ & $<0.001$ & 0.504 \\
\hline
\end{tabular}

The parameters were expressed as mean values \pm standard deviations. LA-A-left atrial maximum transverse diameter; LA-B-left atrial anteroposterior diameter; LAVR-left atrial-vertebral ratio; LV-D1-Left ventricular basal dimension; LV-D2-left ventricular mid cavity dimension; LV-D3left ventricular apical-basal dimension; RA-A-right atrial major dimension; RA-B-right atrial minor dimension; RV-D1-right ventricular basal dimension; RV-D2-right ventricular mid cavity dimension; RV-D3-right ventricular apical-basal dimension

In phase $45 \%$, the cut-off value of LA-A was $77.57 \mathrm{~mm}$ with $36.4 \%$ sensitivity, $97.6 \%$ specificity, $57.1 \%$ positive prediction value, $94.6 \%$ negative prediction value. The cut-off value of LA-B was $37.12 \mathrm{~mm}$ with $90.9 \%$ sensitivity, $37.6 \%$ specificity, $13.2 \%$ positive prediction value, $98.4 \%$ negative prediction value. And the combined diagnosis of LA-A and LA-B had $45.5 \%$ sensitivity, $98.4 \%$ specificity, $71.4 \%$ positive prediction value, $95.4 \%$ negative prediction value. Besides, LAVR had 1.845 cut-off value with $81.8 \%$ sensitivity, $52.4 \%$ specificity, $13 \%$ positive prediction value, $97.1 \%$ negative prediction value.

\subsection{LV parameters in different cardiac cycles}

Table 2 list the mean \pm sd of LV chamber dimensions (LVD1, LVD2, LVD3) in different cardiac phases, Fig. 2A shows the change trends. The maximum and minimum values of LV dimensions were observed in the $5 \%$ phase and $35-45 \%$ phase, respectively. The maximum values of LV-D1, LV-D2, and LV-D3 were $45.09 \pm 4.16 \mathrm{~mm}, 50.44 \pm 4.73 \mathrm{~mm}$, and $77.36 \pm 8.36 \mathrm{~mm}$, respectively. The variation of LV-D1, LV-D2, and LV-D3 in cardiac cycles was of statistical significance. Of these diameters, LV-D1 showed the most stable changes during the cardiac cycle.

Results from univariate analysis revealed that body surface area (BSA), heart rate (HR), LV-myocardial mass (LVMM), smoking, and diabetes were significantly related to LV-D2 and LV-D3 (Table 3). Multivariate analysis demonstrated that HR and smoking were potential indicators of LV-D2, and that HR and LVMM were potential indicators of LV-D3. One unit change in HR was associated with 0.187 unit decrease in the LV-D2. The average LV$\mathrm{D} 2$ of smokers is $2.830 \mathrm{~mm}$ higher than that of non-smokers. The other variables were not significantly related to LV-D2. One unit change in HR was associated with 0.164 unit decease in the LV-D3, while one unit change in LVMM was associated with 0.124 unit increase in the LV-D3. 
Table 3

the univariate analysis of potential related factors for LVD2 and LVD3

\begin{tabular}{|c|c|c|c|c|c|c|}
\hline Characteristics & LV-D2 & $t$ & p-value & LVD3 & $t$ & p-value \\
\hline Age & 0.021 & 0.482 & 0.631 & -0.175 & -3.256 & 0.001 \\
\hline BMI & 0.149 & 0.804 & 0.423 & -0.082 & -0.346 & 0.730 \\
\hline BSA & 8.500 & 2.750 & 0.007 & 14.096 & 3.642 & $<0.001$ \\
\hline HR & -0.222 & -4.130 & $<0.001$ & -0.231 & -3.288 & 0.001 \\
\hline LVMM & 0.058 & 3.242 & 0.002 & 0.140 & 6.965 & $<0.001$ \\
\hline $\begin{array}{l}\text { Smoker } \\
\text { No } \\
\text { Yes }\end{array}$ & $\begin{array}{l}41.29 \pm 9.30 \\
45.34 \pm 8.63\end{array}$ & 3.631 & $<0.001$ & $\begin{array}{l}68.58 \pm 10.16 \\
72.00 \pm 9.37\end{array}$ & 2.352 & 0.020 \\
\hline $\begin{array}{l}\text { Drinker } \\
\text { No } \\
\text { Yes }\end{array}$ & $\begin{array}{l}41.96 \pm 9.03 \\
43.63 \pm 10.44\end{array}$ & 1.231 & 0.221 & $\begin{array}{l}68.87 \pm 10.16 \\
71.88 \pm 9.28\end{array}$ & 1.838 & 0.068 \\
\hline $\begin{array}{l}\text { Hypertension } \\
\text { No } \\
\text { Yes }\end{array}$ & $\begin{array}{l}42.14 \pm 9.43 \\
42.56 \pm 8.98\end{array}$ & 0.402 & 0.688 & $\begin{array}{l}69.47 \pm 10.42 \\
69.19 \pm 9.11\end{array}$ & -0.175 & 0.861 \\
\hline $\begin{array}{l}\text { Diabetes } \\
\text { No } \\
\text { Yes }\end{array}$ & $\begin{array}{l}42.62 \pm 9.34 \\
39.52 \pm 8.62\end{array}$ & -2.040 & 0.044 & $\begin{array}{l}69.68 \pm 10.15 \\
67.28 \pm 9.29\end{array}$ & -1.231 & 0.221 \\
\hline $\begin{array}{l}\text { Dyslipidemia } \\
\text { No } \\
\text { Yes }\end{array}$ & $\begin{array}{l}41.88 \pm 9.28 \\
43.37 \pm 9.30\end{array}$ & 1.315 & 0.191 & $\begin{array}{l}69.34 \pm 10.15 \\
69.56 \pm 9.87\end{array}$ & 0.156 & 0.876 \\
\hline
\end{tabular}

Table 4 lists the mean \pm sd of LV wall thickness at the basal, mid, and apical segments in different cardiac phases. Figure $2 \mathrm{~B}-\mathrm{C}$ shows the change trend graph of LV wall thickness. The LV wall thickness achieved the minimum at the phase of 95-5\%, and the maximum at 35-45\%. The maximum values of the interventricular septum (Septum), anterior (Ant), antero-lateral (Ant-Lat), infero-lateral (Post-Lat), and inferior (Inf) at the basal segment were $10.80 \pm 1.47 \mathrm{~mm}, 11.82 \pm 2.46 \mathrm{~mm}, 11.87 \pm 2.49 \mathrm{~mm}, 12.03 \pm 2.32 \mathrm{~mm}$, and $10.75 \pm 2.10 \mathrm{~mm}$, respectively. The variation of Ant and Ant-Lat in cardiac cycles were of statistical significance. The maximum values of Septum, Ant, Ant-Lat, Post-Lat, and inferior Inf at the mid segment were 11.15 $\pm 1.98 \mathrm{~mm}, 10.22 \pm 2.53 \mathrm{~mm}, 10.75 \pm 2.88 \mathrm{~mm}, 10.93 \pm 2.56 \mathrm{~mm}$, and $11.89 \pm 2.39 \mathrm{~mm}$, respectively. The variation of Septum, Ant, Ant-Lat, Post-Lat, Inf in cardiac cycles was of statistical significance. The maximum values of Septum, Ant, Ant-Lat, and Inf at the apical segment were $9.42 \pm 2.19 \mathrm{~mm}$, $10.83 \pm 2.31 \mathrm{~mm}, 10.60 \pm 2.14 \mathrm{~mm}$, and $9.15 \pm 2.03 \mathrm{~mm}$, respectively. The variation of Septum, Ant, Ant-Lat, and Inf in the cardiac cycles were of statistical significance. In the three segments, the wall changes of the basal segment were the more stable than middle and apical segments. 
Table 4

Left ventricular wall thickness in different cardiac cycles

\begin{tabular}{|c|c|c|c|c|c|c|c|c|c|c|c|c|c|c|}
\hline & Basal & & & & & Mid & & & & & Apical & & & \\
\hline Phase & Septum & Ant & $\begin{array}{l}\text { Ant- } \\
\text { Lat }\end{array}$ & $\begin{array}{l}\text { Post- } \\
\text { Lat }\end{array}$ & $\operatorname{lnf}$ & Septum & Ant & $\begin{array}{l}\text { Ant- } \\
\text { Lat }\end{array}$ & $\begin{array}{l}\text { Post- } \\
\text { Lat }\end{array}$ & $\operatorname{lnf}$ & Septum & Ant & $\begin{array}{l}\text { Ant- } \\
\text { Lat }\end{array}$ & $\operatorname{lnf}$ \\
\hline $5 \%$ & $\begin{array}{l}7.76 \pm \\
1.09\end{array}$ & $\begin{array}{l}7.04 \\
\pm 1.30\end{array}$ & $\begin{array}{l}7.38 \\
\pm 1.12\end{array}$ & $\begin{array}{l}7.55 \\
\pm 1.19\end{array}$ & $\begin{array}{l}7.52 \\
\pm 1.31\end{array}$ & $\begin{array}{l}7.93 \pm \\
1.59\end{array}$ & $\begin{array}{l}6.08 \\
\pm 1.14\end{array}$ & $\begin{array}{l}6.53 \\
\pm 1.04\end{array}$ & $\begin{array}{l}6.63 \\
\pm 1.03\end{array}$ & $\begin{array}{l}7.69 \\
\pm 1.30\end{array}$ & $\begin{array}{l}5.93 \pm \\
1.46\end{array}$ & $\begin{array}{l}6.89 \\
\pm 1.36\end{array}$ & $\begin{array}{l}6.61 \\
\pm 1.24\end{array}$ & $\begin{array}{l}5.54 \\
\pm 1.15\end{array}$ \\
\hline $15 \%$ & $\begin{array}{l}8.29 \pm \\
1.18\end{array}$ & $\begin{array}{l}7.45 \\
\pm 1.46\end{array}$ & $\begin{array}{l}7.82 \\
\pm 1.42\end{array}$ & $\begin{array}{l}7.73 \\
\pm 1.35\end{array}$ & $\begin{array}{l}7.69 \\
\pm 1.33\end{array}$ & $\begin{array}{l}8.48 \pm \\
1.80\end{array}$ & $\begin{array}{l}6.53 \\
\pm 1.14\end{array}$ & $\begin{array}{l}7.02 \\
\pm 1.23\end{array}$ & $\begin{array}{l}7.18 \\
\pm 1.27\end{array}$ & $\begin{array}{l}8.18 \\
\pm 1.40\end{array}$ & $\begin{array}{l}6.52 \pm \\
1.79\end{array}$ & $\begin{array}{l}7.66 \\
\pm 1.52\end{array}$ & $\begin{array}{l}7.34 \\
\pm 1.57\end{array}$ & $\begin{array}{l}5.88 \\
\pm 1.34\end{array}$ \\
\hline $25 \%$ & $\begin{array}{l}9.60 \pm \\
1.33\end{array}$ & $\begin{array}{l}9.31 \\
\pm 2.23\end{array}$ & $\begin{array}{l}9.28 \\
\pm 1.98\end{array}$ & $\begin{array}{l}9.34 \\
\pm 1.88\end{array}$ & $\begin{array}{l}8.90 \\
\pm 1.80\end{array}$ & $\begin{array}{l}9.82 \pm \\
2.02\end{array}$ & $\begin{array}{l}8.19 \\
\pm 1.90\end{array}$ & $\begin{array}{l}8.51 \\
\pm 2.06\end{array}$ & $\begin{array}{l}8.79 \\
\pm 1.86\end{array}$ & $\begin{array}{l}10.04 \\
\pm 2.08\end{array}$ & $\begin{array}{l}7.79 \pm \\
2.03\end{array}$ & $\begin{array}{l}9.04 \\
\pm 1.94\end{array}$ & $\begin{array}{l}8.94 \\
\pm 1.84\end{array}$ & $\begin{array}{l}7.44 \\
\pm 1.83\end{array}$ \\
\hline $35 \%$ & $\begin{array}{l}10.61 \pm \\
1.45\end{array}$ & $\begin{array}{l}11.82 \\
\pm 2.46\end{array}$ & $\begin{array}{l}11.61 \\
\pm 2.59\end{array}$ & $\begin{array}{l}11.41 \\
\pm 2.37\end{array}$ & $\begin{array}{l}10.07 \\
\pm 1.94\end{array}$ & $\begin{array}{l}11.15 \pm \\
1.98\end{array}$ & $\begin{array}{l}10.22 \\
\pm 2.53\end{array}$ & $\begin{array}{l}10.75 \\
\pm 2.84\end{array}$ & $\begin{array}{l}10.93 \\
\pm 2.56\end{array}$ & $\begin{array}{l}11.89 \\
\pm 2.39\end{array}$ & $\begin{array}{l}9.42 \pm \\
2.19\end{array}$ & $\begin{array}{l}10.79 \\
\pm 2.16\end{array}$ & $\begin{array}{l}10.60 \\
\pm 2.14\end{array}$ & $\begin{array}{l}9.15 \\
\pm 2.03\end{array}$ \\
\hline $45 \%$ & $\begin{array}{l}10.80 \pm \\
1.47\end{array}$ & $\begin{array}{l}11.19 \\
\pm 2.63\end{array}$ & $\begin{array}{l}11.87 \\
\pm 2.49\end{array}$ & $\begin{array}{l}12.03 \\
\pm 2.32\end{array}$ & $\begin{array}{l}10.75 \\
\pm 2.10\end{array}$ & $\begin{array}{l}10.75 \pm \\
1.98\end{array}$ & $\begin{array}{l}9.48 \\
\pm 2.28\end{array}$ & $\begin{array}{l}10.75 \\
\pm 2.88\end{array}$ & $\begin{array}{l}10.79 \\
\pm 2.70\end{array}$ & $\begin{array}{l}11.40 \\
\pm 2.47\end{array}$ & $\begin{array}{l}9.39 \pm \\
2.07\end{array}$ & $\begin{array}{l}10.83 \\
\pm 2.31\end{array}$ & $\begin{array}{l}10.51 \\
\pm 2.30\end{array}$ & $\begin{array}{l}8.73 \\
\pm 2.16\end{array}$ \\
\hline $55 \%$ & $\begin{array}{l}10.24 \pm \\
1.58\end{array}$ & $\begin{array}{l}9.05 \\
\pm 2.09\end{array}$ & $\begin{array}{l}9.56 \\
\pm 3.31\end{array}$ & $\begin{array}{l}9.35 \\
\pm 2.01\end{array}$ & $\begin{array}{l}9.65 \\
\pm 4.13\end{array}$ & $\begin{array}{l}9.29 \pm \\
1.88\end{array}$ & $\begin{array}{l}7.45 \\
\pm 1.45\end{array}$ & $\begin{array}{l}7.93 \\
\pm 1.79\end{array}$ & $\begin{array}{l}8.00 \\
\pm 2.06\end{array}$ & $\begin{array}{l}9.04 \\
\pm 2.10\end{array}$ & $\begin{array}{l}7.28 \pm \\
1.78\end{array}$ & $\begin{array}{l}8.71 \\
\pm 3.62\end{array}$ & $\begin{array}{l}8.40 \\
\pm 2.01\end{array}$ & $\begin{array}{l}6.95 \\
\pm 1.89\end{array}$ \\
\hline $65 \%$ & $\begin{array}{l}9.22 \pm \\
1.34\end{array}$ & $\begin{array}{l}8.23 \\
\pm 1.57\end{array}$ & $\begin{array}{l}8.49 \\
\pm 1.45\end{array}$ & $\begin{array}{l}8.95 \\
\pm 1.51\end{array}$ & $\begin{array}{l}8.71 \\
\pm 1.49\end{array}$ & $\begin{array}{l}8.63 \pm \\
1.66\end{array}$ & $\begin{array}{l}6.67 \\
\pm 1.18\end{array}$ & $\begin{array}{l}7.06 \\
\pm 1.24\end{array}$ & $\begin{array}{l}7.13 \\
\pm 1.26\end{array}$ & $\begin{array}{l}8.47 \\
\pm 1.52\end{array}$ & $\begin{array}{l}6.61 \pm \\
1.66\end{array}$ & $\begin{array}{l}7.64 \\
\pm 1.55\end{array}$ & $\begin{array}{l}7.47 \\
\pm 1.50\end{array}$ & $\begin{array}{l}6.35 \\
\pm 1.49\end{array}$ \\
\hline $75 \%$ & $\begin{array}{l}8.85 \pm \\
1.32\end{array}$ & $\begin{array}{l}7.93 \\
\pm 1.44\end{array}$ & $\begin{array}{l}8.19 \\
\pm 1.19\end{array}$ & $\begin{array}{l}8.86 \\
\pm 3.78\end{array}$ & $\begin{array}{l}8.57 \\
\pm 1.44\end{array}$ & $\begin{array}{l}8.45 \pm \\
1.68\end{array}$ & $\begin{array}{l}6.47 \\
\pm 1.15\end{array}$ & $\begin{array}{l}6.91 \\
\pm 1.17\end{array}$ & $\begin{array}{l}7.07 \\
\pm 1.31\end{array}$ & $\begin{array}{l}8.47 \\
\pm 1.55\end{array}$ & $\begin{array}{l}6.53 \pm \\
1.68\end{array}$ & $\begin{array}{l}7.57 \\
\pm 1.66\end{array}$ & $\begin{array}{l}7.35 \\
\pm 1.63\end{array}$ & $\begin{array}{l}6.30 \\
\pm 1.49\end{array}$ \\
\hline $85 \%$ & $\begin{array}{l}8.59 \pm \\
1.19\end{array}$ & $\begin{array}{l}7.73 \\
\pm 1.45\end{array}$ & $\begin{array}{l}8.26 \\
\pm 3.62\end{array}$ & $\begin{array}{l}8.31 \\
\pm 1.37\end{array}$ & $\begin{array}{l}8.28 \\
\pm 1.33\end{array}$ & $\begin{array}{l}8.27 \pm \\
1.62\end{array}$ & $\begin{array}{l}6.29 \\
\pm 1.10\end{array}$ & $\begin{array}{l}6.73 \\
\pm 1.08\end{array}$ & $\begin{array}{l}7.16 \\
\pm 3.83\end{array}$ & $\begin{array}{l}8.17 \\
\pm 1.43\end{array}$ & $\begin{array}{l}6.38 \pm \\
1.67\end{array}$ & $\begin{array}{l}7.35 \\
\pm 1.63\end{array}$ & $\begin{array}{l}7.16 \\
\pm 1.62\end{array}$ & $\begin{array}{l}6.07 \\
\pm 1.48\end{array}$ \\
\hline $95 \%$ & $\begin{array}{l}8.02 \pm \\
1.22\end{array}$ & $\begin{array}{l}7.03 \\
\pm 1.14\end{array}$ & $\begin{array}{l}7.66 \\
\pm 1.12\end{array}$ & $\begin{array}{l}7.85 \\
\pm 1.26\end{array}$ & $\begin{array}{l}7.61 \\
\pm 1.27\end{array}$ & $\begin{array}{l}7.81 \pm \\
1.63\end{array}$ & $\begin{array}{l}5.93 \\
\pm 0.95\end{array}$ & $\begin{array}{l}6.38 \\
\pm 0.98\end{array}$ & $\begin{array}{l}6.54 \\
\pm 0.99\end{array}$ & $\begin{array}{l}7.72 \\
\pm 1.28\end{array}$ & $\begin{array}{l}5.89 \pm \\
1.48\end{array}$ & $\begin{array}{l}6.87 \\
\pm 1.53\end{array}$ & $\begin{array}{l}6.65 \\
\pm 1.41\end{array}$ & $\begin{array}{l}5.60 \\
\pm 1.21\end{array}$ \\
\hline$t$ & -1.892 & -5.248 & -2.879 & -1.571 & -0.596 & -7.641 & -8.179 & -7.447 & -6.332 & -6.541 & -6.940 & -6.388 & -7.132 & -5.404 \\
\hline $\begin{array}{l}\mathrm{p}- \\
\text { value }\end{array}$ & 0.059 & $\begin{array}{l}< \\
0.001\end{array}$ & 0.004 & 0.117 & 0.551 & $<0.001$ & $\begin{array}{l}< \\
0.001\end{array}$ & $\begin{array}{l}< \\
0.001\end{array}$ & $\begin{array}{l}<.001 \\
0.00\end{array}$ & $\begin{array}{l}< \\
0.001\end{array}$ & $<0.001$ & $\begin{array}{l}< \\
0.001\end{array}$ & $\begin{array}{l}<.001 \\
0.00\end{array}$ & $\begin{array}{l}<.001 \\
0 .\end{array}$ \\
\hline
\end{tabular}

\subsection{RA parameters in different cardiac cycles}

Table 2 list the mean \pm sd of the right atrial major dimension (RA-A) and the mean values of the RA minor dimension (RA-B) in different cardiac phases, while Fig. $2 \mathrm{C}$ shows the change trends. For RA-A and RA-B, the maximum and minimum values were observed in the $45 \%$ phase and $95 \%$ phase, respectively. The maximum values of RA-A and RA-B were $45.58 \pm 5.18 \mathrm{~mm}$ and $49.68 \pm 5.26 \mathrm{~mm}$, respectively. The variations of RA-B in cardiac cycles were of statistical significance, while the variations of RA-A in cardiac cycles were non-significant. Compared with RA-A, RA-B changes more stably during the cardiac cycle.

\subsection{RV parameters in different cardiac cycles}

Table 2 list mean \pm sd of the RV chamber dimensions (RVD1, RVD2, RVD3) in different cardiac phases. The maximum and minimum values of RV dimensions were observed in the $95-5 \%$ phase and 35-45\% phase, respectively. The maximum values of RV-D1, RV-D2, and RV-D3 were $42.13 \pm 5.19$ $\mathrm{mm}, 31.83 \pm 5.69 \mathrm{~mm}$ and $74.35 \pm 7.96 \mathrm{~mm}$, respectively. The variation of RV-D1 and RV-D2 in cardiac cycles was of statistical significance, while the variation of RV-D3 in cardiac cycles was non-significant. Among these diameters, RV-D2 showed the most stable changes during the cardiac cycle.

In addition, the comparison of clinical characteristics and cardiac parameters between male and female subjects revealed significant discrepancy in individual characteristics, including age, BSA, HR, LVMM, smoking, drinking, and diabetes. These differences were also significantly related to gender. Upper and lower reference limits were higher in men than in women. Therefore, we generated cardiac parameters reference ranges on cardiac CT in different cardiac phases for both genders for our population in supplemental material.

\subsection{Reproducibility}

Inter- and intraobserver ICCs were determined randomly among 126 patients. Interobserver ICCs of 0.913 for LA-A, 0.920 for LA-B, 0.935 for LV-D1, 0.926 for LV-D2, 0.917 for LV-D3, 0.925 for RA-A, 0.921 for RA-B, 0.936 for RV-D1, 0.956 for RV-D2 and 0.941 for RV-D3 were obtained. Also, intraobserver ICCs of 0.902 for LA-A, 0.912 for LA-B, 0.928 for LV-D1, 0.915 for LV-D2, 0.903 for LV-D3, 0.901 for RA-A, 0.907 for RA-B, 0.924 for RV-D1, 0.917 for RV-D2 and 0.916 for RV-D3 were obtained.

\section{Discussion}


This is the first study to conduct a detailed dynamic evaluation of the cardiac chamber dimensions and wall thickness with the reconstruction of ten cardiac phases in whole cardiac cycle. This allows us to choose the correct cardiac phase for measuring the maximum diameter required to predict the occurrence and recurrence of cardiac disease. The dimensions of the cardiac chamber and wall thickness changes dynamically in different cardiac phases and match the physiological phase of that cycle[16]. We also found that LA-B, LV-D1, RA-B and RV-D2 were more stable than other diameters in the cardiac cycle. Therefore, we believe that the four diameters are most suitable for evaluating the condition of the heart on noncardiac CT images.

LA size is a marker of the severity and chronicity of diastolic dysfunction[17]. These LA diameters measured on a conventional axial cross-section can be used for detecting of patients with possible LA enlargement on routine chest CTA, prompting confirmatory evaluation with echocardiography. Stoyan Popkirov proposed that Coronary CTA should be performed in patients with acute stroke, and that the measurement of chest echocardiography was not accurate, indicating the importance of CT measurement of LA enlargement[18]. Atrial fibrillation (AF) is the most common cardiac arrhythmia encountered in clinical practice. LA enlargement is associated with atrial remodeling, which is associated with recurrence of atrial fibrillation after radiofrequency catheter ablation (RFCA). LA-B is an independent predictor of AF recurrence. While some studies have shown that the larger or smaller the LA-B has the higher the prevalence of AF recurrence[19-21]. We found that the LA-B value varies greatly between $29.54 \pm 4.43$ $\mathrm{mm}$ and $37.70 \pm 4.40$, rather than staying constant throughout the whole cardiac cycle, which might mean that changes of LA-B value in the cardiac cycle should be taken into account when evaluating radiofrequency ablation in patients with atrial fibrillation by using the LA-B maximum value. Selecting appropriate phase for LA-B measurement is more conducive to the choosing of appropriate ablation method and the prediction of AF recurrence. Cryoballoon (CB) and radiofrequency (RF) are common methods of ablation. Given the short procedure time and efficacy, $\mathrm{CB}$ ablation may be recommended for patients without enlarged LA, while RF would be more appropriate in large LA size [22]. MDCT of the pulmonary veins and LA provides the necessary anatomic information for successful CB and RF catheter ablation [23]. At the same time, choosing phase $35 \%$ and $5 \%$ to measure LA-B is of great significance, and the LA volume can also be obtained. LAssVR is a simple new measure directly scaling the left atrial diameter to the anthropomorphic characteristics of the patient. With the use of ROC analysis, cardiac CT measurements that provided a optimal test characteristics for identifying LAE by echocardiography as the reference standard were determined.

LV is an integral part of the heart's pumping function. Historically, linear dimension of LV was measured at the base of the LV in the long-axis view. However, the base of the LV does not reflect the true (maximal) diameter of the ellipsoid mode, leading to underestimation of chamber size[24]. Measurement of linear dimensions at the midventricular level (LV-D2) better reflects the ellipsoid geometry of the LV cavity and provides a more accurate estimate of LV mass and size as compared with the traditionally recommended basal level[13]. In addition, the LV contains obliquely-oriented myofibers superficially, longitudinally-oriented myofibers in the subendocardium, and predominantly circular fibers in between. This contributes to the more complex movement of the LV, including torsion, translation, rotation, and thickening[25]. It is difficult to comprehensively evaluate the size of the chamber using a single diameter measurement method. Therefore, in this study, we measured three dimensions of LV in different cardiac phases. Multivariate analysis demonstrated that HR and smoking are potential indicators of LV-D2. HR and LVMM are potential indicators of LV-D3.

Cardiac remodeling is an important pathological process in the occurrence and development of various cardiovascular diseases such as essential hypertension and cardiomyopathy, including changes in the cardiac chamber dimensions and the wall thickness. The ventricular septum is the central structure of both ventricles. The helical ventricular band model explains the close relationship between biventricular function and ventricular septum[26]. Perhaps this is one of the reasons why myocardial wall hypertrophy first appears as ventricular septal hypertrophy. In this study, the ventricular septum thickness was significantly related to age and hypertension. Therefore, we believe that blood pressure and age are synergistically involved in the occurrence and development of left ventricular remodeling.

There is increasing evidence that RA enlargement is an outcome predictor in various cardiac conditions[27]. To date, diameters and areas measured in the apical four-chamber view are the only recommended methods for assessing RA size[28].

RV function is an independent determinant of clinical status and prognosis in a number of pathologies, but its accurate quantification remains a challenge. As compared with LV, the unique features of the RV are its complex geometry, its wider range of loading conditions, and its greater heterogeneity of regional function. RV failure is usually caused by left heart dysfunction. Both conditions coexist[29]. Ventricular interdependence is not only manifested in function, but also in size, which is more obvious in diastole.

\section{Limitations}

The most obvious limitation of this study was the radiation dose generated by using CT. However, the radiation dose is $3.47 \pm 0.68 \mathrm{mSv}$, which is lower than the radiation protection requirement of X-ray computed tomography. For much lower radiation, scanning voltage and current have been adjusted for body weight and body mass index. And CT is necessary for evaluating coronary artery disease. It can observe not only coronary artery disease but also the heart and make a comprehensive diagnosis of disease. The advantages outweigh the disadvantages for patients with indications, such as coronary heart disease. Moreover, it was a cross-sectional study, and only patients with clear image qualities were evaluated. The free wall of the right ventricle is only $3-5 \mathrm{~mm}$, which can be almost ignored. Simultaneously, we adjusted the window width and window level to make the heart cavity more recognizable and easier to measure. Our research requires further follow-up.

\section{Conclusion}


This study provides applicable quantification cardiac CT cardiac chamber dimensions and LV wall thickness reference ranges in different cardiac phases. Reconstruction and measurement performed in the maximum phase using cardiac CT could result in a more accurate diameter in cardiac disease assessment during the varied cardiac cycle.

\section{Declarations}

\section{Funding}

Not applicable.

\section{Compliance with ethical standards}

\section{Conflict of interest}

All authors have nothing to declare.

\section{Ethics approval}

This study was conducted in accordance with the the Declaration of Helsinki (as was revised in 2013), and an application for the exemption of patients' informed consent was approved by the Institutional Review Board of our hospital, due to the retrospective nature of the study. So all written informed consent forms are waived.

\section{Authors' contributions}

LW and JZ collected data, and were major contributors in writing the manuscript, DC and YD analyzed data, JC provided methods of this study and reviewed the manuscript. All authors read and approved the final manuscript.

\section{Availability of data and materials}

The datasets used and/or analysed during the current study are available from the corresponding author on reasonable request.

\section{Code availability}

Not applicable.

\section{Consent for publication}

Not applicable.

\section{References}

1. Takemoto Y, Barnes ME, Seward JB, Lester SJ, Appleton CA, Gersh BJ, Bailey KR, Tsang TS(2005) Usefulness of left atrial volume in predicting first congestive heart failure in patients $>$ or $=65$ years of age with well-preserved left ventricular systolic function. Am J Cardiol 96:832-836

2. Moller JE, Hillis GS, Oh JK, Seward JB, Reeder GS, Wright RS, Park SW, Bailey KR, Pellikka PA(2003) Left atrial volume: a powerful predictor of survival after acute myocardial infarction. Circulation 107:2207-2212

3. Stolzmann P, Scheffel H, Trindade PT, Plass AR, Husmann L, Leschka S, Genoni M, Marincek B, Kaufmann PA, Alkadhi H(2008) Left Ventricular and Left Atrial Dimensions and Volumes: Comparison Between Dual-Source CT and Echocardiography. Invest Radiol 43:284-289

4. Lin FY, Devereux RB, Roman MJ, Meng J, Jow VM, Jacobs A, Weinsaft JW, Shaw LJ, Berman DS, Callister TQ, Min JK(2008) Cardiac chamber volumes, function, and mass as determined by 64-multidetector row computed tomography: mean values among healthy adults free of hypertension and obesity. JACC Cardiovasc Imaging 1:782-786

5. Ikenouchi T, Inaba O, Takamiya T, Inamura Y, Sato A, Matsumura Y, Sato H, Hirakawa A, Takahashi Y, Goya M, Sasano T, Nitta J(2021) The impact of left atrium size on selection of the pulmonary vein isolation method for atrial fibrillation: Cryoballoon or radiofrequency catheter ablation. Am Heart J 231:82-92

6. Hundley WG, Bluemke DA, Finn JP, Flamm SD, Fogel MA, Friedrich MG, Ho VB, Jerosch-Herold M, Kramer CM, Manning WJ, Patel M, Pohost GM, Stillman AE, White RD, Woodard PK (2010) ACCF/ACR/AHA/NASCI/SCMR 2010 expert consensus document on cardiovascular magnetic resonance: a report of the American College of Cardiology Foundation Task Force on Expert Consensus Documents. Circulation 121:2462-2508

7. Juergens KU, Grude M, Maintz D, Fallenberg EM, Wichter T, Heindel W, Fischbach R (2004) Multi-detector row CT of left ventricular function with dedicated analysis software versus MR imaging:initial experience. Radiology 230:403-410

8. Yamamuro M, Tadamura E, Kubo S, Toyoda H, Nishina T, Ohba M, Hosokawa R, Kimura T, Tamaki N, Komeda M, Kita T, Konishi J (2005) Cardiac functional analysis with multi-detector row CT and segmental reconstruction algorithm: comparison with echocardiography, SPECT, and MR imaging. Radiology 234:381-390 
9. Nevsky G, Jacobs JE, Lim RP, Donnino R, Babb JS, Srichai MB (2011) Sex-specific normalized reference values of heart and great vessel dimensions in cardiac CT angiography. AJR Am J Roentgenol 196:788-794

10. Roberts WT, Bax JJ, Davies LC (2008) Cardiac CT and CT coronary angiography: technology and application. Heart 94:781-792

11. Eifer DA, Nguyen ET, Thavendiranathan P, Hanneman K (2018) Diagnostic Accuracy of Sex-Specific Chest CT Measurements Compared With Cardiac MRI Findings in the Assessment of Cardiac Chamber Enlargement. AJR Am J Roentgenol 211:993-999

12. Baque-Juston M, Volondat M, Fontas E, Roger C, Brunner P, Padovani B, Chevallier P (2016) Left atrio-vertebral ratio:A new computed-tomography measurement to identify left atrial dilation. Eur J Radiol 85:255-260

13. Chetrit M, Roujol S, Picard MH, Timmins L, Manning WJ, Rudski LG, Levine RA, Afilalo J (2019) Optimal Technique for Measurement of Linear Left Ventricular Dimensions. J Am Soc Echocardiogr 32:476-483

14. Kou S, Caballero L, Dulgheru R, Voilliot D, De Sousa C, Kacharava G, Athanassopoulos GD, Barone D, Baroni M, Cardim N, Gomez De Diego JJ, Hagendorff A, Henri C, Hristova K, Lopez T, Magne J, De La Morena G, Popescu BA, Penicka M, Ozyigit T, Rodrigo Carbonero JD, Salustri A, Van De Veire N, Von Bardeleben RS, Vinereanu D, Voigt JU, Zamorano JL, Donal E, Lang RM, Badano LP, Lancellotti P (2014) Echocardiographic reference ranges for normal cardiac chamber size: results from the NORRE study. Eur Heart J Cardiovasc Imaging 15:680-690

15. Rose M, Rubal B, Hulten E, Slim JN, Steel K, Furgerson JL, Villines TC, Slim AM (2014) Chamber dimensions and functional assessment with coronary computed tomographic angiography as compared to echocardiography using American Society of Echocardiography guidelines. SAGE Open Med. doi:10.1177/2050312114522789

16. Deng W, Yang ZG, Peng LQ, Dong ZH, Chu ZG, Wang QL (2010) Morphological and dynamic features of normal mitral valve evaluated by dualsource computed tomography. Int J Cardiol 145:633-636

17. Tsang TS, Barnes ME, Gersh BJ, Bailey KR, Seward JB (2002) Left atrial volume as a morphophysiologic expression of left ventricular diastolic dysfunction and relation to cardiovascular risk burden. Am J Cardiol 90:1284-1289

18. Popkirov S (2020) Left Atrial Enlargement Could Be Detected on Extended Computed Tomography Angiography Within Initial Stroke Assessment. JAMA Neurol 77:134

19. Wang Q, Zhuo C, Shang Y, Zhao J, Chen N, Lv N, Huang Y, Zheng L, Lai J, Han J, Shu Z(2019) U-Shaped Relationship Between Left Atrium Size on Echocardiography and 1-Year Recurrence of Atrial Fibrillation After Radiofrequency Catheter Ablationã囚冈- Prognostic Value Study. Circ J 83:14631471

20. Berruezo A, Tamborero D, Mont L, Benito B, Tolosana JM, Sitges M, Vidal B, Arriagada G, Méndez F, Matiello M, Molina I, Brugada J(2007) Preprocedural predictors of atrial fibrillation recurrence after circumferential pulmonary vein ablation. Eur Heart J 28:836-841

21. den Uijl DW, Delgado V, Bertini M, Tops LF, Trines SA, van de Veire NR, Zeppenfeld K, Schalij MJ, Bax JJ(2011) Impact of left atrial fibrosis and left atrial size on the outcome of catheter ablation for atrial fibrillation. Heart 97:1847-1851

22. Ikenouchi T, Inaba O, Takamiya T, Inamura Y, Sato A, Matsumura Y, Sato H, Hirakawa A, Takahashi Y, Goya M, Sasano T, Nitta J(2021) The impact of left atrium size on selection of the pulmonary vein isolation method for atrial fibrillation: Cryoballoon or radiofrequency catheter ablation. Am Heart J 231:82-92

23. Lacomis JM, Wigginton W, Fuhrman C, Schwartzman D, Armfield DR, Pealer KM (2003) Multi-detector row CT of the left atrium and pulmonary veins before radio-frequency catheter ablation for atrial fibrillation. Radiographics. doi:10.1148/rg.23si035508

24. Aurigemma GP, Silver KH, McLaughlin M, Mauser J, Gaasch WH (1994) Impact of chamber geometry and gender on left ventricular systolic function in patients $>60$ years of age with aortic stenosis. Am J Cardiol 74:794-798

25. Haddad F, Hunt SA, Rosenthal DN, Murphy DJ (2008) Right Ventricular Function in Cardiovascular Disease, Part I: Anatomy, Physiology, Aging, and Functional Assessment of the Right Ventricle. Circulation 117:1436-1448

26. Buckberg GD, RESTORE Group (2006) The ventricular septum: the lion of right ventricular function, and its impact on right ventricular restoration. Eur J Cardiothorac Surg Suppl 1:S272-S278

27. Cioffi G, de Simone G, Mureddu G, Tarantini L, Stefenelli C (2007) Right atrial size and function in patients with pulmonary hypertension associated with disorders of respiratory system or hypoxemia. Eur J Echocardiogr 8:322-331

28. Peluso D, Badano LP, Muraru D, Bianco LD, Cucchini U, Kocabay G (2013) Right atrial size and function assessed with three-dimensional and speckle-tracking echocardiography in 200 healthy volunteers. Eur Heart J Cardiovasc Imaging 14:1106 - 1114, Kovàcs A, Casablanca S, Iliceto S

29. Schwarz K, Singh S, Dawson D, Frenneaux MP (2013) Right ventricular function in left ventricular disease: pathophysiology and implications. Heart Lung Circ 22:507-511

\section{Figures}




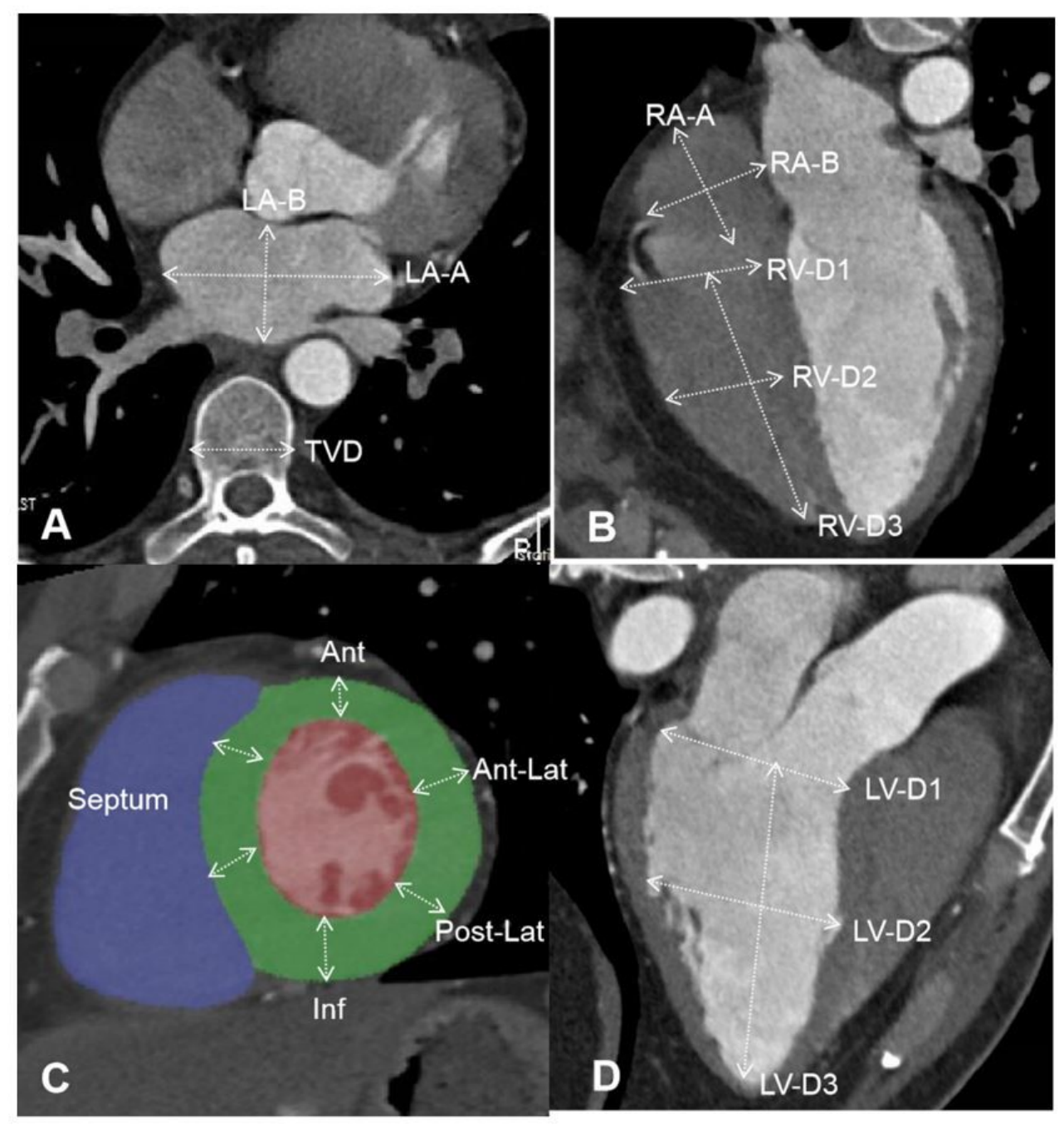

Figure 1

Measurement of the cardiac chamber dimension and wall thickness A:LA-A, LA-B were to be traced manually on an image showing the right inferior pulmonary vein insertion(thus avoiding the left auricle), in a plane parallel to the posterior vertebral wall, TVD was measured at the same level. B:RA-A, RA-B, RVD1, RVD2, and RVD3 were measured in the apical four-chamber MPR view. C:The left ventricular wall thickness at the basal, mid and apical segment were measured in the short-axis view of LV. D:LVD1, LVD2, and LVD3 were measured in the long-axis view of LV. (LA-A,left atrial maximum transverse diameter; LA-B,left atrial antero-posterior diameter; TVD, the thoracic vertebral diameter; RA-A, right atrial major dimension; RA-B, right atrial minor dimension; RVD1, right ventricular basal dimension; RVD2,right ventricular mid cavity dimension; RVD3, right ventricular apical-basal dimension; Septum,interventricular septum; Ant, anterior; Ant-Lat,antero-lateral; Post-Lat,infero-lateral; Inf,inferior; LVD1, left ventricular basal dimension; LVD2,left ventricular mid cavity dimension; LVD3, left ventricular apical-basal dimension) 

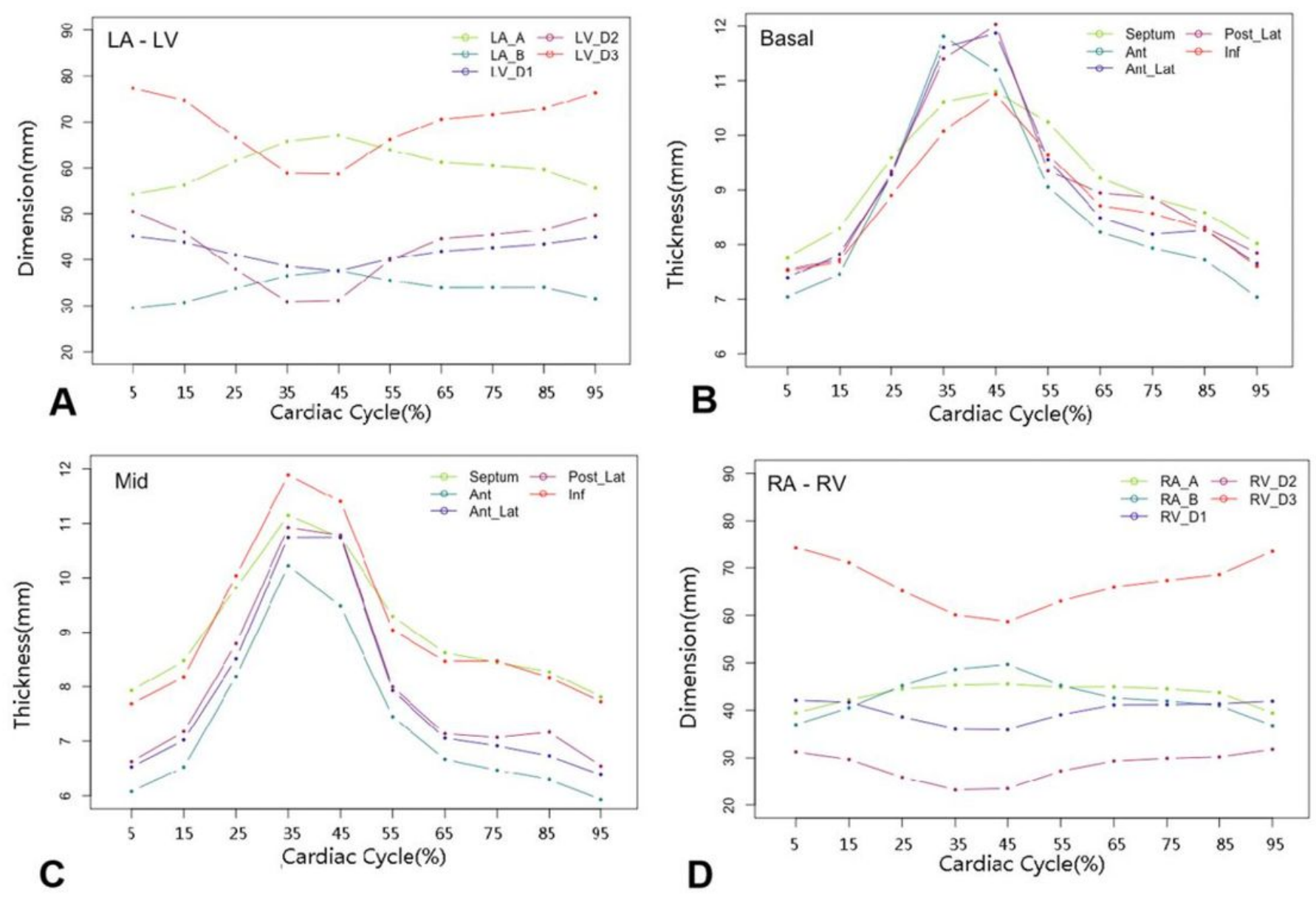

Figure 2

The change trends with cardiac phases of (A)LA and LV, (B) the LV wall thickness of basal, (C)the LV wall thickness of mid, (D)RA and RV. (Abbreviations as in Fig. 1) 